\title{
Diagnostic suite used for magnetohydrodynamics equilibrium reconstruction on the PEGASUS toroidal experiment (abstract) ${ }^{a}$ )
}

A. C. Sontag, ${ }^{\text {b) }}$ G. D. Garstka, R. J. Fonck, R. J. Schooff, T. A. Thorson, and K. L. Tritz University of Wisconsin, Madison, Madison, Wisconsin 53706

(Presented on 19 June 2000)

Magnetic equilibrium reconstruction on the PEGASUS toroidal experiment is a crucial tool to determine macroscopic plasma parameters, such as geometry, $l_{i}, \beta_{t}$, and $q_{\psi}$. These parameters are tightly coupled to the plasma shape due to the very high toroidicity in PEGASUS where $A \approx 1.1-1.3$. A systematic scan of model plasma parameters in a magnetic equilibrium code has been employed to determine an acceptable array of magnetic diagnostics for accurately characterizing the plasma equilibrium. The magnetic diagnostics used include a poloidal array of magnetic pickup coils and flux loops along with a Rogowski loop for the toroidal plasma current. A $270 \mathrm{GHz} \mu$ wave interferometer for line averaged density in conjunction with spectroscopic temperature estimates provide a central pressure constraint. Visible images of the plasma provide constraints on the plasma size and location. A one-dimensional SXR camera is being developed to provide a measurement of the magnetic axis location. A time evolving current filament model and wall flux loops are used to determine the induced currents flowing in the continuous, resistive vacuum vessel wall. The ability of the equilibrium reconstruction code to reproduce model equilibria using this diagnostic set provides a quantitative measure of the accuracy of these equilibrium reconstructions. A Monte Carlo analysis with Gaussian noise added to the model data tests the robustness of this technique. A comparison of the model equilibria with the reconstructions obtained using noisy data is shown.

(C) 2001 American Institute of Physics. [DOI: 10.1063/1.1323472]

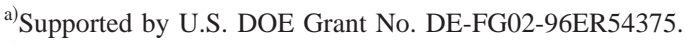

b) Present address: 1500 Engineering Drive, Madison, WI 53706; electronic mail: sontag@cae.wisc.edu 